This item was submitted to Loughborough's Research Repository by the author.

Items in Figshare are protected by copyright, with all rights reserved, unless otherwise indicated.

\title{
Shared-control for a rear-wheel drive car: Dynamic environments and disturbance rejection
}

PLEASE CITE THE PUBLISHED VERSION

https://doi.org/10.1109/thms.2017.2693231

PUBLISHER

(C) Institute of Electrical and Electronics Engineers (IEEE)

VERSION

AM (Accepted Manuscript)

LICENCE

CC BY-NC-ND 4.0

\section{REPOSITORY RECORD}

Jiang, Jingjing, and Alessandro Astolfi. 2017. "Shared-control for a Rear-wheel Drive Car: Dynamic

Environments and Disturbance Rejection”. Loughborough University. https://hdl.handle.net/2134/36751. 


\title{
Shared-Control for a Rear-Wheel Drive Car: Dynamic Environments and Disturbance Rejection
}

\author{
Jingjing Jiang ${ }^{1}$ and Alessandro Astolfi ${ }^{2}$
}

\begin{abstract}
This paper studies the shared-control problem for the kinematic model of a group of rear-wheel drive cars in a (possibly) dynamic (i.e. time-varying) environment. The design of the shared-controller is based on measurements of distances to obstacles, angle differences and the human input. The sharedcontroller is used to guarantee the safety of the car when the driver behaves "dangerously". Formal properties of the closedloop-system with the shared-controller are presented through a Lyapunov-like analysis. In addition, we consider uncertainties in the dynamics and prove that the shared-controller is able to help the driver drive the car safely even in the presence of disturbances. Finally, the effectiveness of the controller is verified by two case studies: traffic at a junction and at a roundabout.
\end{abstract}

\section{NOMENCLATURE}

\begin{tabular}{|c|c|}
\hline$\epsilon$ & a sufficiently small positive constant \\
\hline$\theta$ & heading angle of the car \\
\hline$\theta_{e}$ & $\begin{array}{l}\text { difference between the heading angle and } \\
\text { the reference angle }\end{array}$ \\
\hline$\theta_{r}$ & reference heading angle \\
\hline$\phi$ & $\begin{array}{l}\text { steering angle, i.e. the wheeling angle } \\
\text { between the front-wheel and the heading } \\
\text { direction of the car }\end{array}$ \\
\hline$\phi_{r}$ & reference steering angle \\
\hline$\Omega_{s}, \Omega_{h}$ & $\begin{array}{l}\Omega \text {-limit set of the s-closed-loop system, h- } \\
\text { closed-loop system }\end{array}$ \\
\hline$\Pi_{\mathcal{R}_{s}}\left(\Omega_{h}\right)$ & projection of $\Omega_{h}$ into the set $\mathcal{R}_{s}$ \\
\hline$D$ & $\begin{array}{l}\text { distance to the obstacle along the current } \\
\text { forward direction }\end{array}$ \\
\hline$d_{1}$ & $\begin{array}{l}\text { distance to the obstacle along the direction } \\
\text { of the reference forward velocity }\end{array}$ \\
\hline$d_{2 l}, d_{2 r}$ & $\begin{array}{l}\text { distance to the obstacles on the left-hand } \\
\text { side, right-hand side, orthogonal to the } \\
\text { direction of the reference forward velocity }\end{array}$ \\
\hline$d_{d 1}, d_{d 2}$ & reference trajectory for the states $d_{1}, d_{2}$ \\
\hline$d_{r 1}, d_{r 2}$ & $\begin{array}{l}\text { feasible reference trajectory for the state } d_{1} \text {, } \\
d_{2}\end{array}$ \\
\hline$\hat{d}_{1}, \hat{d}_{2}$ & $\begin{array}{l}\text { minimum value of } d_{1}, d_{2} \text {, to guarantee that } \\
\text { the car operates in "safe" conditions }\end{array}$ \\
\hline$d_{x}, d_{y}, d_{\theta}, d_{\phi}$ & $\begin{array}{l}\text { disturbance on the dynamics of states } x, y \text {, } \\
\theta, \phi\end{array}$ \\
\hline $\bar{d}_{1}, \bar{d}_{2}, \bar{d}_{3}, \bar{d}_{4}$ & bound of the variable $d_{x}, d_{y}, d_{\theta}, d_{\phi}$ \\
\hline$e$ & \\
\hline$k$ & sharing function \\
\hline$l$ & $\begin{array}{l}\text { en the middle of the rear- } \\
\text { front-axle }\end{array}$ \\
\hline
\end{tabular}

\footnotetext{
${ }^{1}$ J. Jiang is with the Department of Electrical and Electronic Engineering, Imperial College London, UK, E-mail: jingjing.jiang10@imperial.ac.uk

${ }^{2} \mathrm{~A}$. Astolfi is with the Dept. of Electrical and Electronic Engineering, Imperial College London, London, SW7 2AZ, UK and the DICII, University of Roma "Tor Vergata", Via del Politecnico 1, 00133 Rome, Italy, E-mail: a.astolfi@imperial.ac.uk
}

$p=[x, y]^{T}$
$\mathcal{P}_{a}$
$\mathcal{R}_{s}$
$\mathcal{R}_{h}$
$\mathcal{R}_{d}$
$u_{f}$
$u_{h}$
$u_{s}$
$v_{r}$
$w$

Cartesian coordinates of the middle of the rear-axle admissible Cartesian configuration set "safe" set "hysteresis" set "dangerous" set feedback control inputs driver inputs shared-control inputs reference forward velocity distance between the center of the two rear wheels

Index Terms-Shared-control, Control constraints, Nonlinear control, Driver assistance

\section{INTRODUCTION}

Automobile has become the most popular means of transport since the 20th century. Nowadays, even though we have several modes of transport, such as high-speed rails, undergrounds and aircrafts, cars are still the core means to transport human beings and goods from one place to another, especially for short journeys. According to the latest report, more than 70 million cars have been sold worldwide in 2015 and the market increases year by year [1]. Unfortunately, along with the increase use of the automobile, the road traffic accidents caused by cars are the major cause of fatality [2]. Over one million people are killed by traffic accidents every year. Therefore, the enhancement of road safety becomes a critical issue not only for governments but also for car producers and researchers.

There are two ways to increase the safety of the vehicle. One is named as "passive safety": it relies on the use of seat belts, air bags and passenger safety cells. These equipments are used to reduce the effects of accidents. The other safety system is termed as "active safety": this helps avoid accidents (for example the anti-lock braking system). It is obvious that active safety systems are preferable and more desirable. However, they are more difficult to design and implement. That is why the design of an "active safe" controller for cars has attracted researchers' attentions in recent years.

[3] has used a decentralized $H_{\infty}$ controller to minimize energy consumption within a bounded tracking error and to avoid obstacles, while [4] has provided a comparison of different control methods based on PI and MPC (Model Predictive Control) and highlighted the key advantages and disadvantages for each method. Another auto-driving scheme is made up of two steps: finding out the pool of all safe behaviours and 
then picking the most appropriate one from that pool [5]. These control designs are all based on the solution of an optimization problem, in which the choice of the optimization time horizon is crucial. Other control methods, such as sliding mode [6], [7], [8], machine learning [9], [10] and fuzzy logics [11], [12], [13], have also been explored and implemented. In addition, [14] has proposed a navigation algorithm based on 9 subsystems generated from step responses in various operating conditions.

The situation becomes even more complicated when taking human driver into consideration. As stated in [15] there are various ways for the human to interact with the system. In some systems the human generates the strategy to be performed and then passes it to the system to be executed automatically. For example, planetary robots designed for exploration are able to execute the "command" (such as move 5 meters forward) sent by the ground controller automatically. Another popular class of systems with humans in the control loop are modern aircrafts. They are mainly controlled by the pilot during taking off and landing. But the control authority is passed to the "autopilot" if the aircraft is flying smoothly until unexpected situations happen, when the control authority is regained by the pilot. Besides airplanes, "Google car" is another example which aims to liberate the driver from the control loop but allows him/her to take charge in emergencies or "bad" situations. However, our approach which describes how human driver interacts with the car is opposite to "Google Cars". The control authority is held by the human driver most of the time to let him/her enjoy the "fun" of driving and it is gained by the feedback controller only if the driver behaves "dangerously". Furthermore, [16] has introduced a shared controller, based on the "active area" where the vehicle is allowed to drive, to combine the driver and the local controller. The method is based on a constrain optimization scheme which minimizes the deviation from the given rider input. Another common way to deal with the shared-control problem is to let the human operator supervise the operation and take critical decisions in high-risk situations [17], [18]. Furthermore, [19] has introduced the concept of "capture set" in which brakes should be used to avoid collisions. Based on the calculation of this set, the feedback controller is active and overrides the human's command only to prevent collisions. Therefore the idea of the shared-control algorithm is similar to the one presented in this paper. Yet, we control not only the forward velocity (longitudinal control) but also the angular velocity of the front wheels. In addition, significant research has been done to model human behaviours [20], [21], [22]. However, due to the complexity of human being, more work is still needed to model it. In order to get rid of this, [23] provides a shared-controller based on the judgment of the current situation and proves that the established shared-control law for the kinematic model of a car works for any human input: if the human behaves "dangerously" then the feedback controller is active and seizes the control authority; if the human behaves "safely" then the car responds only to the human behaviour. Furthermore, the car with the shared-control is able to reach any bound of the feasible region safely given that the set of feasible Cartesian positions is defined by a group of linear inequalities. This paper extends the results given in [23] to cases in which disturbances are considered. In addition, it studies the shared-control problem for the car in a non-predefined and possibly dynamic environment. Formal mathematical proof have been given to highlight that the safety of the car is guaranteed with the established shared-control law.

This paper is organized as follows. Section II describes the model we study and formulates the shared-control problem. This is followed by some assumptions and definitions which are used in the rest of the paper. The design of the sharedcontroller for the kinematic model of a rear-wheel drive car in a time-varying environment without measurements of absolute positions is given in Section III, in which formal properties of the closed-loop system are presented. To deal with uncertainties in system modeling, Section IV studies the shared-control for the kinematic model of a car in the presence of bounded disturbances. Section V gives two case studies to illustrate how the shared-control algorithm works. Finally, some conclusions and suggestions for the future work are given in Section VI.

\section{PROBLEM STATEMENT}

The paper studies the kinematic model of a rear-wheel driving car, the dynamics of which can be described by the equations

$$
\begin{aligned}
\dot{x} & =v_{s} \cos \theta, \\
\dot{y} & =v_{s} \sin \theta, \\
\dot{\theta} & =\frac{v_{s} \tan \phi}{l}, \\
\dot{\phi} & =\omega_{s},
\end{aligned}
$$

where $v_{s}$ and $\omega_{s}$ are the shared-control inputs, describing the linear velocity of the car and the angular velocity of the frontwheels, respectively.

Definition 1: The shared-control input $u_{s}=\left[v_{s}, \omega_{s}\right]^{T}$ is a combination of human input $u_{h}=\left[v_{h}, \omega_{h}\right]^{T}$, denoted as $h$-control, and the feedback control input $u_{f}=\left[v_{f}, \omega_{f}\right]^{T}$, denoted as $f$-control. It is defined as

$$
\begin{aligned}
v_{s} & =(1-k) v_{f}+k v_{h}, \\
\omega_{s} & =(1-k) \omega_{f}+k \omega_{h},
\end{aligned}
$$

where $k$, denoted as sharing function, quantifies how the control authority is shared between the driver and the feedback controller. In addition, we use the name $s$-closed-loop and $h$ closed-loop to denote the closed-loop system described by (1)(2) with $k \in[0,1]$ and $k=1$, respectively.

We study the system with either full state feedback or output feedback. In the cases in which state feedback is available we assume that absolute positions are measurable. On the other hand, in the cases with output feedback we assume that our measurements include the distance to the obstacle along the direction of the current forward velocity $D$, the angular difference between the actual and the reference heading angle 


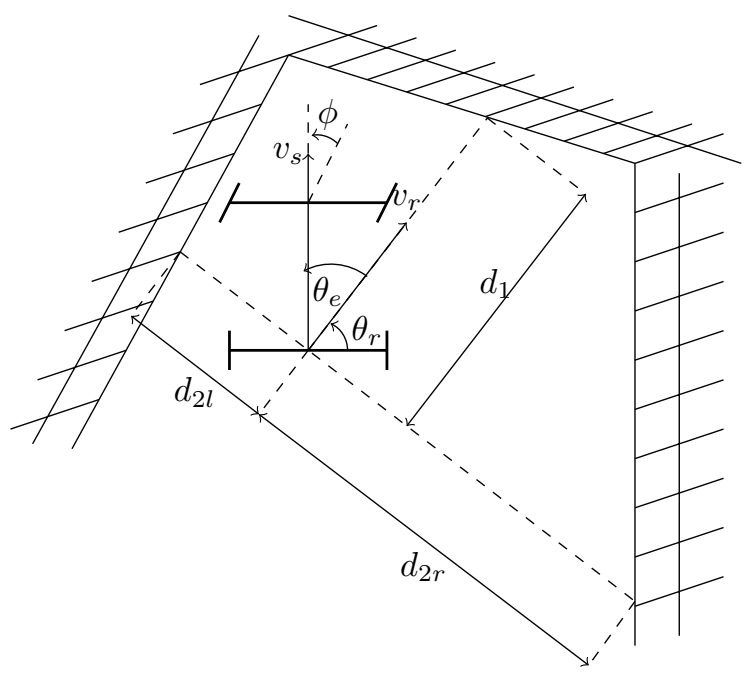

Fig. 1: Definitions of $d_{1}, d_{2 l}, d_{2 r}, \theta_{e}$ and $\phi$ (shadowed region: unfeasible region, $v_{r}$ : reference forward velocity, $\theta_{r}$ : reference forward angle, $v_{s}$ : actual forward velocity)

$\theta_{e}$, the steering angle $\phi$ and the distances to the obstacles along and orthogonal to the reference forward direction $d_{1}$ and

$$
d_{2}= \begin{cases}d_{2 l} \quad & \text { if }\left|\log \frac{d_{2 l}-\hat{d}_{2}}{d_{r_{2 l}}-\hat{d}_{2}}\right| \leq\left|\log \frac{d_{2 r}-\hat{d}_{2}}{d_{r_{2 r}}-\hat{d}_{2}}\right|, \\ d_{2 r} & \text { if }\left|\log \frac{d_{2 l}-\hat{d}_{2}}{d_{r_{2 l}}-\hat{d}_{2}}\right|>\left|\log \frac{d_{2 r}-\hat{d}_{2}}{d_{r_{2 r}}-\hat{d}_{2}}\right|,\end{cases}
$$

where $d_{2 l}$ and $d_{2 r}$ denote the distances to the obstacles on the left-hand side and the right-hand side orthogonal to the direction of the reference forward velocity, respectively (see Figure 1). Note that $d_{2}$ is defined as above to guarantee the continuity of the variable $z_{2}$, defined in (5), when switching between $d_{2}=d_{2 l}$ and $d_{2}=d_{2 r}$. In addition, $\hat{d}_{2}$ is defined as the minimum value of the variable $d_{2}$ to guarantee the "safety" of the car.

Definition 2: The definition of omega limit set, denoted as $\Omega$ limit set, is given as follows. In trajectory tracking cases the definition of $\Omega$-limit set is the same as that given in [24]. On the other hand, in cases in which there is no given reference signal to track, let $x=\Phi\left(t, x_{0}, u(t)\right)$ be the solution of the differential equation $\dot{x}=f(x, u(t))$ with initial state $x=x_{0}$, where $f \in \mathbb{C}^{k}\left(M, \mathbb{R}^{n}\right)$, with $k \geq 1$ and $M$ an open subset of $\mathbb{R}^{n}$. Consider $x_{0} \in M$. The omega limit set of $x$ with the given initial state $x_{0}$, denoted as $\Omega(x)$, is the set of points $y \in M$ such that $\Phi\left(t, x_{0}, u(t)\right)=y$.

Suppose $\mathcal{P}_{a}(t) \subset \mathbb{R}^{2}$ is a closed and compact set describing the admissible Cartesian configurations (i.e. $(x(t), y(t)) \in$ $\mathcal{P}_{a}(t)$ for all $\left.t \geq 0\right)$ for the system (1) and $u_{h}=\left[v_{h}, \omega_{h}\right]^{T}$ is a given h-control. The shared-control problem for the kinematic model of a rear-wheel driving car can be formulated as follows.

Given the system (1), an h-control and an admissible configuration set $\mathcal{P}_{a}(t)$ which is such that the initial condition $(x(0), y(0)) \in \mathcal{P}_{a}$, find (if possible)

- an f-control $u_{f}$;
- a sharing function $k$;

- a safe set $\mathcal{R}_{s}\left(v_{h}, t\right) \triangleq \mathcal{P}_{a}(t) \times \mathcal{H}_{s} \times \mathcal{A}_{s} \subset \mathcal{P}_{a}(t) \times \mathcal{H} \times$ $\mathcal{A} \triangleq \mathcal{R}\left(v_{h}, t\right)$

where $\mathcal{H}\left(\mathcal{H}_{s}\right)$ and $\mathcal{A}\left(\mathcal{A}_{s}\right)$ are the sets of heading angles (safe heading angles) and wheeling angles (safe wheeling angles), respectively, such that the s-closed-loop system (1)-(2) has the following properties.

P1) The set $\mathcal{R}\left(v_{h}, t\right)$ is forward invariant, i.e. $(x(t), y(t)) \in$ $\mathcal{P}_{a}$ for all $t \geq 0$.

P2)

$$
\Omega_{s}= \begin{cases}\Omega_{h} & \text { if } \Omega_{h} \subset \mathcal{R}_{s}\left(v_{h}, t\right), \\ \Pi_{\mathcal{R}_{s}}\left(\Omega_{h}\right) & \text { if } \Omega_{h} \not \subset \mathcal{R}_{s}\left(v_{h}, t\right),\end{cases}
$$

where $\Pi_{\mathcal{R}_{s}}\left(\Omega_{h}\right)$ will be defined in Section III-A and IV-A without and with measurements of absolute positions, respectively.

P3) $u_{s}(t)=u_{h}(t)$ for all positive $t$ such that the state of the system belongs to $\mathcal{R}_{s}\left(v_{h}, t\right)$.

An intuitive description of the sets $\mathcal{H}_{s}$ and $\mathcal{A}_{s}$ is given as follows. For any fixed $v_{h}$ and any $(x, y) \in \mathcal{P}_{a}$, the sets $\mathcal{H}_{s}(t)$ and $\mathcal{A}_{s}(t)$ are the sets of all possible heading angles and frontwheel angles such that the car cannot hit the boundary of $\mathcal{P}_{a}$ within a short period of time. In other words, the car will not hit any obstacles within a short period of time as long as the system states belong to $\mathcal{R}_{s}\left(v_{h}, t\right)$ at time the instant $t$.

If disturbances are considered, modification should be made to P2) as follows:

$$
\begin{cases}\Omega_{s}=\Omega_{h}, & \text { if } \Omega_{h} \subset \mathcal{R}_{s}\left(v_{h}, t\right), \\ \left\|\Omega_{s}-\Omega_{h}\right\|_{2} \text { is bounded, } & \text { if } \Omega_{h} \not \subset \mathcal{R}_{s}\left(v_{h}, t\right),\end{cases}
$$

Assumption 1: The projection of the car in the $(x, y)$-plane is a rectangle with length $l$ and width $w$.

Assumption 2: The admissible Cartesian configuration set for the car is always non-empty, i.e. $\mathcal{P}_{a}(t) \neq \emptyset, \forall t \geq 0$.

Definition 3: The function $h(y(t), x(t), t)=\operatorname{atg}(y(t), x(t), t)$ is a continuous function defined as

$$
\operatorname{atg}(y(t), x(t), t)=\operatorname{atan}(y(t), x(t))+2 c(t) \pi,
$$

where atan is the standard four quadrant arctan function, $c(0)=0$ and

$$
c(t)= \begin{cases}c(t-\delta t)+1, & \text { if } a=-2 \pi \\ c(t-\delta t)-1, & \text { if } a=2 \pi \\ c(t-\delta t), & \text { else }\end{cases}
$$

with $a=\lim _{\delta t \rightarrow 0^{+}} \operatorname{atan}(y(t), x(t))-\operatorname{atan}(y(t-\delta t), x(t-\delta t))$ for all $t>0$.

Note that the definition of the function $\operatorname{atg}(y(t), x(t), t)$ is close to that of the standard four quadrant arctan function $\operatorname{atan}(y(t), x(t))$ except that $\operatorname{atg}(\dot{)}$ is a continuous function and the range of it equals $(-\infty,+\infty)$ instead of $[-\pi, p i)$. 


\section{DESIGN OF THE SHARED-CONTROL WITHOUT MEASUREMENTS OF ABSOLUTE POSITIONS}

Paper [23] presents a shared-control algorithm for a rear-wheel drive car based on the measurements of absolute positions. However, absolute positioning is not always available. This section gives a solution to the shared-control problem stated in Section II without measurements of absolute positions. This contains two steps: design of the feedback controller, in Section III-A, and definition of how the control authority is shared between the driver and the feedback controller, in Section III-B.

\section{A. Design of the Feedback Controller}

According to the definition, $d_{2}=d_{2 l}$ or $d_{2}=d_{2 r}$. If $d_{2}=d_{2 l}$, then the dynamics of the closed-loop system controlled by the feedback controller can be described by the equations

$$
\begin{aligned}
\dot{d}_{1} & =-v_{f} \cos \theta_{e}, \\
\dot{d}_{2} & =-v_{f} \sin \theta_{e}, \\
\dot{\theta}_{e} & =\frac{v_{f} \tan \phi-v_{r} \tan \phi_{r}}{l}, \\
\dot{\phi} & =\omega_{f} .
\end{aligned}
$$

On the other hand, if $d_{2}=d_{2 r}$, then the dynamics of the closed-loop system with the feedback control $u_{f}$ is similar to (3) except that

$$
\dot{d}_{2}=v_{f} \sin \theta_{e},
$$

i.e. $\dot{d}_{2}$ has the opposite sign to the case in which $d_{2}=d_{2 l}$. Without loss of generality we only study the case in which $d_{2}=d_{2 l}$. As stated in Assumption 1, the car can be regarded as a rectangle with sides length $l$ and $m$. Therefore, the position constraints $(x(t), y(t)) \in \mathcal{P}_{a}(t)$ can be rewritten as

$$
d_{i}(t) \geq \hat{d}_{i}
$$

where $\hat{d}_{i}$ is a positive constant for all $i \in\{1,2\}$. One choice for $\hat{d}_{1}$ and $\hat{d}_{2}$ is $\hat{d}_{1}=\hat{d}_{2}=\sqrt{l^{2}+\left(\frac{w}{2}\right)^{2}}$.

Assumption 3: The function $d_{d}(t)=\left[d_{d 1}(t) ; d_{d 2}(t)\right]^{T}$, representing the desired distances to the relative obstacles (the obstacles along and orthogonal to the reference forward direction), is continuous.

Define the variable $z=\left[z_{1}, z_{2}\right]^{T}$ as

$$
z_{i}=\log \frac{d_{i}-\hat{d}_{i}}{d_{r_{i}}-\hat{d}_{i}}, \forall i \in\{1,2\},
$$

where $d_{r_{1}}$ and $d_{r_{2}}$ describe the reference trajectory for the state $d_{1}$ and $d_{2}$, respectively, and are defined as

$$
d_{r_{i}}= \begin{cases}d_{d_{i}}, & \text { if } d_{d_{i}} \geq\left(1-\frac{\sqrt{2}}{2}\right) r+\epsilon+\hat{d}_{i}, \\ \epsilon+\hat{d}_{i}, & \text { if } d_{d_{i}} \leq(1-\sqrt{2}) r+\epsilon+\hat{d}_{i}, \\ m_{i}, & \text { otherwise }\end{cases}
$$

for $i \in\{1,2\}$, with $m_{i}=r+\epsilon+\hat{d}_{i}-$ $\sqrt{r^{2}-\left[(\sqrt{2}-1) r-\epsilon+d_{d_{i}}-\hat{d}_{i}\right]^{2}}$ and a sufficiently

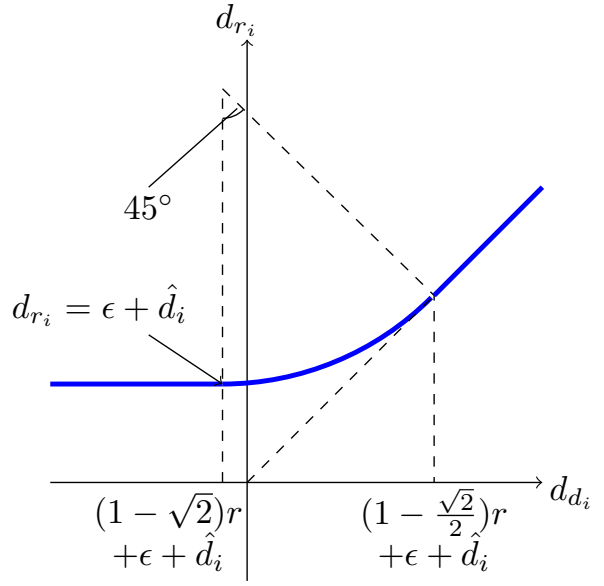

Fig. 2: Graph of the function $d_{r_{i}}$ given by (6) for both $i \in$ $\{1,2\}$.

small positive constant $\epsilon$. Note that the variable $d_{r_{i}}$, instead of $d_{d_{i}}$, is used in the definition of $z_{i}$ because $d_{d_{i}}$ may be unfeasible, i.e. there may exist a positive $t$ such that $d_{i}(t)<\hat{d}_{i}$. However, according to the definition of $d_{r_{i}}$ given in (6), the inequality $d_{r_{i}}(t)>\hat{d}_{i}$ holds for all $t \geq 0$. In addition, (6) guarantees that $d_{r}$ is a smooth signal.

Figure 2 illustrates the definition of $d_{r_{i}}$, from which it is easy to conclude that $d_{r_{i}}$ is a smooth function for $i \in\{1,2\}$. Therefore, $\dot{d}_{r_{1}}$ exists and

$$
v_{r}=-\dot{d}_{r_{1}} .
$$

Consider any point $\left(d_{d_{1}}, d_{d_{2}}, \theta_{d}, \phi_{d}\right)$ in the $\Omega$-limit set of the h-closed-loop system, i.e. $\left(d_{d_{1}}, d_{d_{2}}, \theta_{d}, \phi_{d}\right) \in \Omega_{h}$, the projection of it into the safe subset $\mathcal{R}_{s}$ is defined as

$$
\Pi_{\mathcal{R}_{s}}\left(d_{d_{1}}, d_{d_{2}}, \theta_{d}, \phi_{d}\right)=\left(d_{r_{1}}, d_{r_{2}}, \theta_{d}, \phi_{d}\right)
$$

where $d_{r_{1}}$ and $d_{r_{2}}$ are defined by (6). Hence, the projection of $\Omega_{h}$ into the set $\mathcal{R}_{s}$ is defined by

$$
\Pi_{\mathcal{R}_{s}}\left(\Omega_{h}\right)=\left\{\begin{array}{l|l}
s \in \mathcal{R}_{s} & \begin{array}{c}
s=\Pi_{\mathcal{R}_{s}}\left(d_{d_{1}}, d_{d_{2}}, \theta_{d}, \phi_{d}\right), \\
\forall\left(d_{d_{1}}, d_{d_{2}}, \theta_{d}, \phi_{d}\right) \in \Omega_{h}
\end{array}
\end{array}\right\} .
$$

Using the variable $z$, system (3) can be written as

$$
\begin{aligned}
\dot{z}_{1} & =\frac{v_{r}}{d_{r_{1}}-\hat{d}_{1}}-\frac{v_{f} \cos \theta_{e}}{d_{1}-\hat{d}_{1}}, \\
\dot{z}_{2} & =-\frac{v_{f} \sin \theta_{e}}{d_{2}-\hat{d}_{2}}, \\
\dot{\theta}_{e} & =\frac{v_{f} \tan \phi-v_{r} \tan \phi_{r}}{l}, \\
\dot{\phi} & =\omega_{f} .
\end{aligned}
$$




$$
\begin{gathered}
\mathcal{R}_{s}\left(v_{h}\right)=\left\{\left(d_{1}, d_{2}, \theta_{e}, \phi\right) \in \mathbb{R}^{+} \times \mathbb{R}^{+} \times \mathbb{S} \times \mathbb{S}: v_{h} \leq \frac{1}{b_{2}-D}-\frac{1}{b_{2}} \text { if } D \leq b_{2}\right\} \\
\mathcal{R}_{h}\left(v_{h}\right)=\left\{\begin{array}{c}
\left(d_{1}, d_{2}, \theta_{e}, \phi\right) \in \mathbb{R}^{+} \times \mathbb{R}^{+} \times \mathbb{S} \times \mathbb{S}: \quad \frac{1}{b_{2}-D}-\frac{1}{b_{2}}<v_{h}<\frac{1}{b_{1}-D}-\frac{1}{b_{1}} \text { and } D \leq b_{1} \\
\text { or } v_{h}>\frac{1}{b_{2}-D}-\frac{1}{b_{2}} \text { and } b_{1} \leq D \leq b_{2}
\end{array}\right\} \\
\mathcal{R}_{d}\left(v_{h}\right)=\left\{\left(d_{1}, d_{2}, \theta_{e}, \phi\right) \in \mathbb{R}^{+} \times \mathbb{R}^{+} \times \mathbb{S} \times \mathbb{S}: v_{h} \geq \frac{1}{b_{1}-D}-\frac{1}{b_{1}} \text { and } 0 \leq D \leq b_{1}\right\}
\end{gathered}
$$

Let $^{1}$

$$
\begin{aligned}
\theta_{e}^{*} & =\operatorname{atg}\left(\gamma_{2}\left(d_{2}-\hat{d}_{2}\right) z_{2},\left(d_{1}-\hat{d}_{1}\right)\left(\frac{v_{r}}{d_{r_{1}}-\hat{d}_{1}}+\gamma_{1} z_{1}\right)\right), \\
\alpha & =\sqrt{\left(\gamma_{2}\left(d_{2}-\hat{d}_{2}\right) z_{2}\right)^{2}+\left[\left(d_{1}-\hat{d}_{1}\right)\left(\frac{v_{r}}{d_{r_{1}}-\hat{d}_{1}}+\gamma_{1} z_{1}\right)\right]^{2}}, \\
\phi^{*} & =\operatorname{atg}\left(\begin{array}{c}
\frac{l z_{2}}{d_{2}-\hat{d}_{2}} \cos \frac{\theta_{e}+\theta_{e}^{*}}{2} \operatorname{sinc} \frac{\theta_{e}-\theta_{e}^{*}}{2} \\
-\frac{z_{1}}{d_{1}-\hat{d}_{1}} \sin \frac{\theta_{e}+\theta_{e}^{*}}{2} \operatorname{sinc} \frac{\theta_{e}-\theta_{e}^{*}}{2}, 1 \\
+\frac{v_{r} \tan \phi_{r}}{\alpha}+\frac{l \dot{\theta}_{e}^{*}}{\alpha}
\end{array}\right),
\end{aligned}
$$

where $\gamma_{1}>0, \gamma_{2}>0$.

Consider the Lyapunov function candidate

$$
L\left(z_{1}, z_{2}, \theta_{e}, \phi, t\right)=\frac{1}{2}\left\{\begin{array}{c}
z_{1}^{2}+z_{2}^{2}+\left(\theta_{e}-\theta_{e}^{*}\right)^{2} \\
+\left(\tan \phi-\tan \phi^{*}\right)^{2}
\end{array}\right\},
$$

and select $v_{f}$ and $\omega_{f}$ such that $\dot{L}(t)<0$ for all $t \geq 0$ and $\left(z_{1}, z_{2}, \theta_{e}, \tan \phi\right) \neq\left(0,0, \theta_{e}^{*}, \tan \phi^{*}\right)$. One such a choice is given by

$$
\begin{aligned}
& v_{f}=\alpha, \\
& \omega_{f}=\cos ^{2} \phi\left[\begin{array}{c}
\frac{\dot{\phi}^{*}}{\cos ^{2} \phi^{*}}-\frac{v\left(\theta_{e}-\theta_{e}^{*}\right)}{l} \\
-\gamma_{3}\left(\tan \phi-\tan \phi^{*}\right)
\end{array}\right],
\end{aligned}
$$

where $\gamma_{3}>0$, yielding

$$
\dot{L}=-\gamma_{1} z_{1}^{2}-\gamma_{2} z_{2}^{2}-\gamma_{3}\left(\tan \phi-\tan \phi^{*}\right)^{2} \leq 0 .
$$

Lemma 1: Consider the closed-loop system (3) controlled by the feedback controller (5)-(8)-(10) with $d_{r}$ and $v_{r}$ given by (6) and (7), respectively. Assume the initial position of the car is feasible, i.e. $(x(0), y(0)) \in \mathcal{P}_{a}, d_{1}(0)>\hat{d}_{1}$ and $d_{2}(0)>\hat{d}_{2}$. Then the closed-loop system has the following properties.

- $d_{1}(t)>\hat{d}_{1}, d_{2}(t)>\hat{d}_{2}$ for all $t \geq 0$;

- $\lim _{t \rightarrow \infty}\left(d_{1}(t)-d_{r_{1}}(t)\right)=\lim _{t \rightarrow \infty}\left(d_{2}(t)-d_{r_{2}}(t)\right)=0$;

- $|\phi(t)|<\frac{\pi}{2}$ for all $t \geq 0$.

Proof: The first statement is a trivial consequence of the definition of $z$ given in (5).

\footnotetext{
${ }^{1}$ The function 'atg' is defined in Definition 3.
}

Consider the Lyapunov function (9) and note that $\dot{L}(t) \leq 0$. In addition, $\dot{L} \equiv 0$ implies $\left(z_{1}, z_{2}, \theta_{e}, \tan \phi\right)=\left(0,0, \theta_{e}^{*}, \tan \phi^{*}\right)$. Even though there may be switches between $d_{2}=d_{2 l}$ and $d_{2}=d_{2 r}$, the overall Lyapunov function is continuous due to the definition of $d_{2}$. Hence,

$$
\lim _{t \rightarrow \infty}\left(d_{1}(t)-d_{r_{1}}(t)\right)=\lim _{t \rightarrow \infty}\left(d_{2}(t)-d_{r_{2}}(t)\right)=0 .
$$

Finally, the third property is proved by contradiction. Suppose that there exists a $\bar{t} \geq 0$ such that $\phi(\bar{t})=\frac{\pi}{2}$ or $\phi(\bar{t})=-\frac{\pi}{2}$. Then the Lyapunov function at time instant $\bar{t}$ has a value of $+\infty$, i.e.

$$
\lim _{t \rightarrow \bar{t}} L(t)=+\infty
$$

However, $L(0)$ is bounded and $L(t)$ is decreasing, which implies that $L(t)<L(0)$. This contradicts (11), hence the claim.

\section{B. Shared Control Theorem}

For any given human input $v_{h}$, the safe, hysteresis and dangerous subsets, $\mathcal{R}_{s}, \mathcal{R}_{h}$ and $\mathcal{R}_{d}$, are defined by equations (12) given at the top of this page, where $D$ is the distance to the obstacle along the direction of $v_{h}$ and $b_{2}>b_{1}>0$ are constants selected by the user. The intuition for the set definition is similar to that given in [25]: the situation is "safe" if the car drives towards the obstacle with a bounded top speed (the top speed is a function of the distance to the obstacle); the situation is "dangerous" if (and only if) the car is "close" (i.e. $D \leq b_{1}$ ) to the obstacle and the car drives towards the obstacle with a speed larger than a predefined value (this value is not a constant, but varying with the distance).

The sharing function $k$ can then be defined as

$$
k\left(D, v_{h}\right)= \begin{cases}1, & \left(d_{1}, d_{2}, \theta_{e}, \phi\right) \in \mathcal{R}_{s}\left(v_{h}\right), \\ g, & \left(d_{1}, d_{2}, \theta_{e}, \phi\right) \in \mathcal{R}_{h}\left(v_{h}\right), \\ 0, & \left(d_{1}, d_{2}, \theta_{e}, \phi\right) \in \mathcal{R}_{d}\left(v_{h}\right),\end{cases}
$$

where

$$
g= \begin{cases}1, & \text { if }\left(d_{1}, d_{2}, \theta_{e}, \phi\right) \text { enters } \mathcal{R}_{h}\left(v_{h}\right) \text { from } \mathcal{R}_{s}\left(v_{h}\right), \\ 0, & \text { if }\left(d_{1}, d_{2}, \theta_{e}, \phi\right) \text { enters } \mathcal{R}_{h}\left(v_{h}\right) \text { from } \mathcal{R}_{d}\left(v_{h}\right) .\end{cases}
$$

Finally, the overall shared-control input $u_{s}=\left[v_{s}, \omega_{s}\right]^{T}$ is given by

$$
u_{s}=\left(1-k\left(D, v_{h}\right)\right) u_{f}\left(d, d_{r}, \theta_{e}, \phi, v_{r}\right)+k\left(D, v_{h}\right) u_{h} .
$$


Theorem 1: Consider the kinematic model of a rear-wheel driving car (1) with the shared-control input given by (5)(8)-(10)-(13)-(14). Assume the initial condition is feasible, i.e. $(x(0), y(0)) \in \mathcal{P}_{a}(0), d_{1}(0)>\hat{d}_{1}$ and $d_{2}(0)>\hat{d}_{2}$, and $u_{h}$ is a given h-control. Then there exists $\gamma_{i}>0$, for $i \in\{1,2,3\}$, such that the closed-loop system has the following properties.

1) $d_{1}(t)>\hat{d}_{1}$ and $d_{2}(t)>\hat{d}_{2}$ for all $t \geq 0$;

2) $\Omega_{s}=\Pi_{\mathcal{R}_{s}}\left(\Omega_{h}\right)$;

3) $u_{s}(t)=u_{h}(t)$ for all $t \geq 0$ such that $\left.\left(d_{1}(t), d_{(} t\right), \theta_{e}(t), \phi(t)\right) \in \mathcal{R}_{s}$.

Proof: To begin with, as detailed in Section III-A, the feedback control input $u_{f}$ is such that the configuration of the system stays in the admissible region $\mathcal{P}_{a}(t)$ for all $t \geq 0$. In addition, the definition of $\mathcal{R}_{d}$ indicates that if the state of the system does not belong to the feasible set at the time instant $t=t_{0}$, i.e. $\left(d_{1}\left(t_{0}\right), d_{2}\left(t_{0}\right), \theta_{e}\left(t_{0}\right), \phi\left(t_{0}\right) \notin \mathcal{R}\left(v_{h}, t_{0}\right)\right)$, then there exists $0<\bar{t}<t_{0}$ such that $\left(d_{1}(\bar{t}), d_{2}(\bar{t}), \theta_{e}(\bar{t}), \phi(\bar{t}) \in\right.$ $\mathcal{R}_{d}\left(v_{h}, \bar{t}\right)$. In other words, any trajectory leaving the feasible set $\mathcal{R}$ should enter the dangerous subset where $u_{f}$ is active. Therefore, the set $\mathcal{R}$ is forward invariant and the first property holds.

If $\Omega_{h} \subset \mathcal{R}_{s}$, then the second claim is a consequence of the general results in [26], and of the fact that $\Omega_{h}$ is the $\Omega$-limit set of both the h-closed-loop and system (3) (by assumption, the former, and by equations (5)-(9), the latter). Otherwise, if $\Omega_{h} \not \subset \mathcal{R}_{s}$, Lemma 1 indicates that the $\Omega$-limit set of system (3) is $\Pi_{\mathcal{R}_{s}}\left(\Omega_{h}\right)$. Furthermore, equation (12) indicates that the trajectory of the system enters $\mathcal{R}_{d}$ where the feedback controller is active, hence driving the states of the system back to $\mathcal{R}_{s}$, before leaving the admissible set $\mathcal{R}$. Therefore, property 2) holds.

Finally, Property 3) is a direct consequence of the definition of $u_{s}$.

Remark 1: With $\hat{d}_{1}=\hat{d}_{2}=\sqrt{l^{2}+\left(\frac{w}{2}\right)^{2}}$, the car is unable to stop just next to the boundary of its admissible configuration set. However, this issue can easily be solved by changing values $\hat{d}_{1}$ and $\hat{d}_{2}$ to $l$ and $\frac{w}{2}$, respectively when $\theta_{e}$ is close to 0 .

Remark 2: According to the shared-control law (12)-(13)(14), the feedback controller is active only if the car is "close" to an obstacle. Therefore, $d_{i}$ can be modified by $\hat{d}_{i}=\min \left(d_{i}, \mathcal{B}\right)$, for $i \in\{1,2\}$, where $\mathcal{B}$ is a positive constant selected by the user.

As a result we need to design the feedback controller only for the cases in which $d_{i} \leq \mathcal{B}$. This is very useful in applications, especially in the cases in which the obstacle is exactly parallel or perpendicular to the required direction, i.e. $d_{1}$ or $d_{2}$ equals to infinity. Note that the feedback control design and the shared-control algorithm are based on instantaneous measurements. The safe set at the time instant $t$ is found by calculating all reachable states in the unconstrained space and ensure that there is no collision within a given time horizon.

\section{DISTURBANCE REJECTION}

Until now the model we have studied does not include any uncertainty. To make the model more realistic, we include additive disturbances to the system dynamics, which can therefore be written as

$$
\begin{aligned}
& \dot{x}=v_{s} \cos \theta+d_{x}, \\
& \dot{y}=v_{s} \sin \theta+d_{y}, \\
& \dot{\theta}=\frac{v_{s} \tan \phi}{l}+d_{\theta}, \\
& \dot{\phi}=\omega_{s}+d_{\phi},
\end{aligned}
$$

where $d_{x}, d_{y}, d_{\theta}$ and $d_{\phi}$ are disturbances on the dynamics of $x, y, \theta$ and $\phi$, respectively.

The non-empty constant admissible configuration set of the car is defined by a group of linear inequalities given by

$$
\mathcal{P}_{a}=\left\{p \in \mathbb{R}^{2} \mid S p+T \leq 0\right\},
$$

where $p=[x, y]^{T}, S=\left[s_{1}^{T}, s_{2}^{T}, \ldots, s_{n}^{T}\right]^{T} \in \mathbb{R}^{n \times 2}$ and $T=$ $\left[t_{1}, t_{2}, \ldots, t_{n}\right]^{T} \in \mathbb{R}^{m}$.

Assumption 4: If $m>2$ then the matrices $S$ and $T$ are such that

$$
\operatorname{rank}\left(\left[\begin{array}{l}
s_{r_{1}} \\
\vdots \\
s_{r_{l}}
\end{array}\right]\right)<\operatorname{rank}\left(\left[\begin{array}{ll}
s_{r_{1}} & t_{r_{1}} \\
\vdots & \vdots \\
s_{r_{l}} & t_{r_{l}}
\end{array}\right]\right),
$$

for all $l \in[3, m]$ and $r_{1}, r_{2}, \ldots, r_{l} \in\{1,2, \ldots, m\}$.

Assumption 5: The disturbances are bounded, i.e. $\left|d_{x}\right| \leq \bar{d}_{1}$, $\left|d_{y}\right| \leq \bar{d}_{2},\left|d_{\theta}\right| \leq \bar{d}_{3}$ and $\left|d_{\phi}\right| \leq \bar{d}_{4}$, where $d_{1}, d_{2}, d_{3}$ and $d_{4}$ are positive constants known by the users.

We assume that the above assumptions hold for the rest of this section.

\section{A. Design of the Feedback Controller with the Existence of Disturbances}

Lemma 2: Consider the set $\mathcal{P}_{a}$ given by (16) and assume Assumption 4 holds. Then no more than two constraints are active $^{2}$ at any time instant.

Proof: If $n \leq 2$ then the claim trivially holds. Otherwise, if $n>2$, we prove the lemma by contradiction. Suppose that, for any fixed $x, y, v_{h}$ and $\theta$, three constraints are active. Without loss of generality we assume that these are the first three constraints. Then $\left[s_{1}^{T}, s_{2}^{T}, s_{3}^{T}\right]^{T}\left[x+k v_{h} \cos \theta, y+\right.$ $\left.k v_{h} \sin \theta\right]^{T}+\left[t_{1}, t_{2}, t_{3}\right]^{T}=0$. By Assumption 4 the above equation does not have any solutions, hence the claim.

Lemma 2 indicates that the $n$ different constraints can be partitioned into $N_{c}$ groups and each group contains two

\footnotetext{
${ }^{2}$ For each position $(x, y)$ with velocity $v_{h}$ and heading angle $\theta$, the $j^{\text {th }}$ constraint is active if

$$
\exists k>0: s_{j}\left[x+k v_{h} \cos \theta, y+k v_{h} \sin \theta\right]^{T}+t_{j}=0 .
$$
}


constraints. Note that in general $N_{c} \leq\left(\begin{array}{l}n \\ 2\end{array}\right)$. If two constraints are active at any time instant $t$, then there is only one group of active constraints. However, if only one constraint is active at the time instant $t$, there could be $\eta$ different groups of active constraints, where $1 \leq \eta \leq(n-1)$. For the $i^{\text {th }}$ group of active constraints

$$
m^{i}=\left[m_{1}^{i}, m_{2}^{i}\right]^{T}=S^{i} p+T^{i} \leq 0,
$$

where $T^{i} \in \mathbb{R}^{2}$ and $S^{i}=\left[s_{1}^{i}, s_{2}^{i T}\right]^{T} \in \mathbb{R}^{2 \times 2}$ is an invertible square matrix with $s_{1}^{i}=\left[s_{11}^{i}, s_{12}^{i}\right], s_{2}^{i}=\left[s_{21}^{i}, s_{22}^{i}\right]$, we define the variable $z^{i}=\left[z_{1}^{i}, z_{2}^{i}\right]^{T}$ based on the Cartesian position of the car as

$$
z_{j}^{i}=\log \frac{m_{j}^{i}}{m_{r_{j}}^{i}}, \forall j \in\{1,2\},
$$

where $m_{r}^{i}=\left[m_{r_{1}}^{i}, m_{r_{2}}^{i}\right]^{T}$ is the reference signal relative to $m^{i}$ with $m_{r_{j}}^{i}$ defined by

$$
m_{r_{j}}^{i}= \begin{cases}m_{d_{j}}^{i}, & \text { if } m_{d_{j}}^{i} \leq\left(\frac{\sqrt{2}}{2}-1\right) r-\epsilon \\ -\epsilon, & \text { if } m_{d_{j}}^{i} \geq(\sqrt{2}-1) r-\epsilon, \\ b_{j}^{i}, & \text { otherwise }\end{cases}
$$

where $\gamma>0, \quad m_{d_{j}}^{i}=s_{j}^{i} p_{d}+t_{j}^{i}, \quad b_{j}^{i}=$ $\sqrt{r^{2}-\left[(\sqrt{2}-1) r-\epsilon-m_{d_{j}}^{i}\right]^{2}}-r-\epsilon, \epsilon$ is a sufficiently small positive constant and $p_{d}$ denotes the reference configuration for the variable $p=[x, y]^{T}$. In the trajectory tracking case, the feedback controller and the driver share the same information on the reference trajectory, i.e. $p_{d}$ is known to the feedback controller. On the other hand, in the free-driving case in which there is no given trajectory to track, $p_{d}(t)$ is calculated from $u_{h}(t)$. In other words, the human behaviour is regarded as the reference behaviour by the feedback controller. Note that the mapping $m_{d_{j}}^{i} \rightarrow m_{r_{j}}^{i}$ given by (18) is similar to the mapping $d_{d_{i}} \rightarrow d_{r_{i}}$ defined by (6). In addition, $m_{r_{j}}^{i}$ is a smooth function with all values less than zero for $j=1$ and $j=2$. Therefore $\dot{m}_{r}^{i}$ exists and $\left(S^{i}\right)^{-1} \dot{m}_{r}^{i}=\left[v_{r}^{i} \cos \theta_{r}^{i}, v_{r}^{i} \sin \theta_{r}^{i}\right]^{T}$. Define the variable $q^{i}=\left[q_{1}^{i}, q_{2}^{i}\right]^{T}$ as

$$
q^{i}=\left(S^{i}\right)^{-1} \dot{m}_{r}^{i} .
$$

Then the reference signals $v_{r}^{i}, \theta_{r}^{i}, \phi_{r}^{i}$ and $\omega_{r}^{i}$ can be calculated as

$$
\begin{aligned}
& {\left[x_{r}^{i}, y_{r}^{i}\right]^{T}=\left(S^{i}\right)^{-1}\left(m_{r}^{i}-T^{i}\right), } \theta_{r}^{i}=\operatorname{atg}\left(q_{2}^{i}, q_{1}^{i}\right), \\
& v_{r}^{i}=\sqrt{\left(q_{1}^{i}\right)^{2}+\left(q_{2}^{i}\right)^{2}}, \phi_{r}^{i}=\operatorname{atg}\left(\frac{\dot{\theta}_{r}^{i} l}{v_{r}^{i}}, 1\right), \\
& \omega_{r}^{i}=\dot{\phi}_{r}^{i} .
\end{aligned}
$$

Suppose $\left(x_{d}, y_{d}, \theta_{d}, \phi_{d}\right)$ is a point of the $\Omega$-limit set for the h-closed-loop system, then we define the projection of $\left(x_{d}, y_{d}, \theta_{d}, \phi_{d}\right)$ into the safe subset $\mathcal{R}_{s}$ relative to the $i^{\text {th }}$ group of constraints as

$$
\Pi_{\mathcal{R}_{s}}^{i}\left(x_{d}, y_{d}, \theta_{d}, \phi_{d}\right)=\left(x_{r}^{i}, y_{r}^{i}, \theta_{r}^{i}, \phi_{r}^{i}\right),
$$

where $x_{r}^{i}, y_{r}^{i}, \theta_{r}^{i}$ and $\phi_{r}^{i}$ are given by (19). Therefore, the projection of $\Omega_{h}$ into $\mathcal{R}_{s}$ relative to the $i^{\text {th }}$ group of constraints

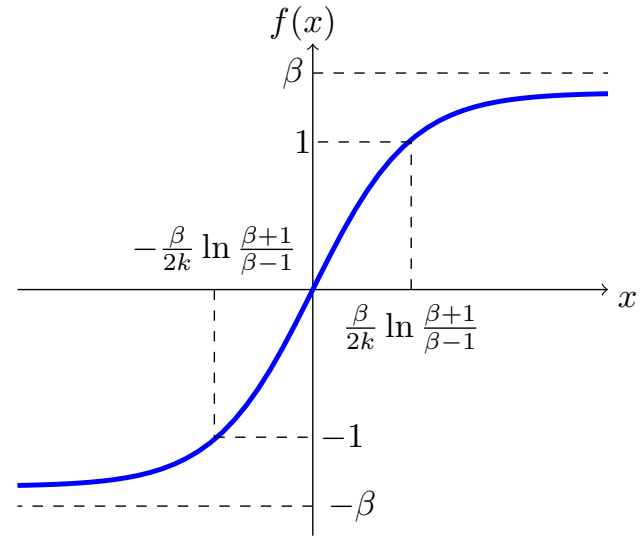

Fig. 3: Graph of the function $f(x)$ given by Definition 4 .

is defined by

$$
\Pi_{\mathcal{R}_{s}}^{i}\left(\Omega_{h}\right)=\left\{\begin{array}{l|l}
s \in \mathcal{R}_{s} & \begin{array}{c}
s=\Pi_{\mathcal{R}_{s}}^{i}\left(x_{d}, y_{d}, \theta_{d}, \phi_{d}\right), \\
\forall\left(x_{d}, y_{d}, \theta_{d}, \phi_{d}\right) \in \Omega_{h}
\end{array}
\end{array}\right\}
$$

We use the new variable $z^{i}$ given by (17) to remove constraints on $m^{i}$. With the use of $z^{i}$, system (15) with the feedback controller $u_{f}^{i}=\left[v_{f}^{i}, \omega_{f}^{i}\right]^{T}$ can be rewritten as

$$
\begin{aligned}
& \dot{z}_{1}^{i}=\frac{s_{11}^{i}\left(v_{f}^{i} \cos \theta+d_{x}\right)+s_{12}^{i}\left(v_{f}^{i} \sin \theta+d_{y}\right)}{e^{z_{1}^{i}} m_{r_{1}}^{i}}-\frac{\dot{m}_{r_{1}}^{i}}{m_{r_{1}}^{i}}, \\
& \dot{z}_{2}^{i}=\frac{s_{21}^{i}\left(v_{f}^{i} \cos \theta+d_{x}\right)+s_{22}^{i}\left(v_{f}^{i} \sin \theta+d_{y}\right)}{e^{z_{2}^{i}} m_{r_{2}}^{i}}-\frac{\dot{m}_{r_{2}}^{i}}{m_{r_{2}}^{i}}, \\
& \dot{\theta}=\frac{v_{f}^{i} \tan \phi}{l}+d_{\theta}, \\
& \dot{\phi}=\omega_{f}^{i}+d_{\phi} .
\end{aligned}
$$

Definition 4: The function $f$ is defined as $f(x)=$ $\beta \tanh \left(\frac{g x}{\beta}\right)$, where $g>0$ and $\beta>1$ are user selected parameters.

According to Definition $4, f$ is a monotonous odd function (displayed in Figure 3) with $f\left(\frac{\beta}{2 k} \ln \frac{\beta+1}{\beta-1}\right)=1$. Note that $\frac{\beta}{2 g} \ln \frac{\beta+1}{\beta-1}>0$ and this can be made sufficiently small by selecting $\beta$ and $k$. In addition, $\lim _{x \rightarrow \infty} f(x)=\beta$ and $\lim _{x \rightarrow-\infty} f(x)=-\beta$.

The back-stepping method is used to find the values of $v_{f}^{i}$ and 
$\omega_{f}^{i}$. The details are explained as follows. Let

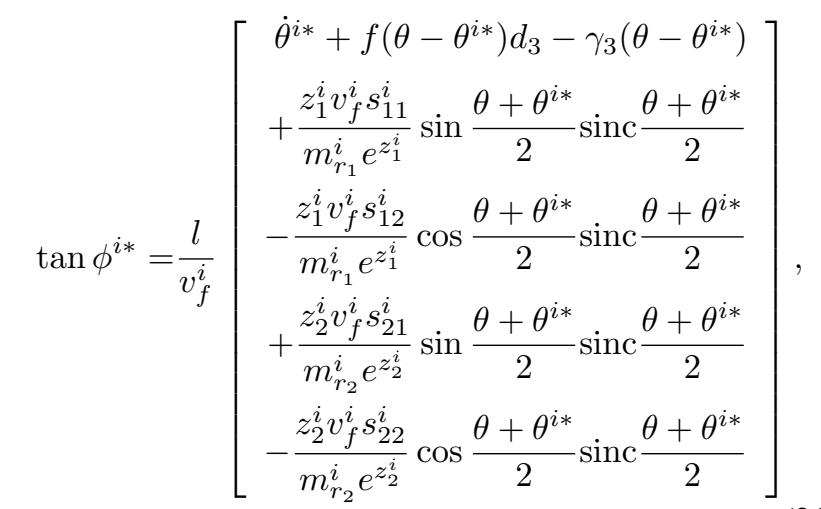

$$
\begin{aligned}
& {\left[\begin{array}{c}
s_{11}^{i} f\left(s_{11}^{i} z_{1}^{i}\right) d_{1}+s_{12}^{i} f\left(s_{12}^{i} z_{1}^{i}\right) d_{2}+e^{z_{1}^{i}}\left(\dot{m}_{r_{1}}^{i}-\gamma_{1} m_{r_{1}}^{i} z_{1}^{i}\right) \\
s_{21}^{i} f\left(s_{21}^{i} z_{2}^{i}\right) d_{1}+s_{22}^{i} f\left(s_{12}^{i} z_{2}^{i}\right) d_{2}+e^{z_{2}^{i}}\left(\dot{m}_{r_{2}}^{i}-\gamma_{2} m_{r_{2}}^{i} z_{2}^{i}\right)
\end{array}\right]} \\
& =S^{i}\left[\begin{array}{c}
v_{f}^{i} \cos \theta^{i *} \\
v_{f}^{i} \sin \theta^{i *}
\end{array}\right]=\left[\begin{array}{c}
\alpha \\
\zeta
\end{array}\right]
\end{aligned}
$$

where $\gamma_{1}, \gamma_{2}$ and $\gamma_{3}$ are three positive constants, the value of which is chosen by the user. Since $S^{i}$ is invertible, we define $\left[q_{1}^{i}, q_{2}^{i}\right]^{T}=\left(S^{i}\right)^{-1}[\alpha, \zeta]^{T}$. Then it is trivial to calculate $v_{f}^{i}$ as

$$
v_{f}^{i}=\sqrt{\left(q_{1}^{i}\right)^{2}+\left(q_{2}^{i}\right)^{2}}, \quad \theta^{i *}=\operatorname{atg}\left(q_{2}^{i}, q_{1}^{i}\right) .
$$

Consider the Lyapunov function candidate relative to the $i^{\text {th }}$ group of constraints given by

$$
L^{i}\left(z_{1}^{i}, z_{2}^{i}, \theta, \phi\right)=\frac{1}{2}\left\{\begin{array}{r}
z_{1}^{i^{2}}+z_{2}^{i^{2}}+\left(\theta-\theta^{i *}\right)^{2} \\
+\left(\tan \phi-\tan \phi^{i *}\right)^{2}
\end{array}\right\},
$$

with $\tan \phi^{i *}$ and $\theta^{i *}$ given by (21a) and (22), respectively. Then the time derivative of $L^{i}$ along the trajectories of the system is calculated as

$$
\begin{aligned}
& \dot{L}^{i}=\frac{z_{1}^{i}}{m_{r_{1}}^{i}}\left(\frac{g_{11}+g_{12}}{e^{z_{1}^{i}}}-\dot{m}_{r_{1}}^{i}\right)+\frac{z_{2}^{i}}{m_{r_{2}}^{i}}\left(\frac{g_{21}+g_{22}}{e^{z_{2}^{i}}}-\dot{m}_{r_{2}}^{i}\right) \\
& +\frac{z_{1}^{i} v_{f}^{i} s_{11}^{i}}{m_{r_{1}}^{i} e^{z_{1}^{i}}}\left(\cos \theta-\cos \theta^{i *}\right)+\frac{z_{1}^{i} v_{f}^{i} s_{12}^{i}}{m_{r_{1}}^{i} e^{z_{1}^{i}}}\left(\sin \theta-\sin \theta^{i *}\right) \\
& +\frac{z_{2}^{i} v_{f}^{i} s_{21}^{i}}{m_{r_{2}}^{i} e^{z_{2}^{i}}}\left(\cos \theta-\cos \theta^{i *}\right)+\frac{z_{2}^{i} v_{f}^{i} s_{22}^{i}}{m_{r_{2}}^{i} e^{z_{2}^{i}}}\left(\sin \theta-\sin \theta^{i *}\right) \\
& +\left(\theta-\theta^{i *}\right)\left(\frac{v_{f}^{i} \tan \phi}{l}+d_{\theta}-\dot{\theta}^{i *}\right)+A+B \\
& +\left(\tan \phi-\tan \phi^{i *}\right)\left(\frac{\omega_{f}^{i}+d_{\phi}}{\cos ^{2} \phi}-\frac{\dot{\phi}^{i *}}{\cos ^{2} \phi^{i *}}\right) \\
& =-\gamma_{1}\left(z_{1}^{i}\right)^{2}-\gamma_{2}\left(z_{2}^{i}\right)^{2}-\gamma_{3}\left(\theta-\theta^{i *}\right)^{2}+A+B+C \\
& +\left(\tan \phi-\tan \phi^{i *}\right)\left[\frac{\omega_{f}^{i}+d_{\phi}}{\cos ^{2} \phi}-\frac{\dot{\phi}^{i *}}{\cos ^{2} \phi^{i *}}+\frac{v_{f}^{i}}{l}\left(\theta-\theta^{i *}\right)\right] \\
& \leq\left(\tan \phi-\tan \phi^{i *}\right)\left[\begin{array}{c}
\frac{\omega_{f}^{i}+\operatorname{sign}\left(\tan \phi-\tan \phi^{i *}\right) d_{4}}{\cos ^{2} \phi} \\
-\frac{\dot{\phi}^{i *}}{\cos ^{2} \phi^{i *}}+\frac{v_{f}^{i}}{l}\left(\theta-\theta^{i *}\right)
\end{array}\right] \\
& +\frac{\gamma_{1}\left(z_{1}^{i}\right)^{2}}{m_{r_{1}}^{i}}+\frac{\gamma_{2}\left(z_{2}^{i}\right)^{2}}{m_{r_{2}}^{i}}-\gamma_{3}\left(\theta-\theta^{i *}\right)^{2}+A+B+C,
\end{aligned}
$$

where

$$
\begin{aligned}
A & =\frac{s_{11}^{i} z_{1}^{i}}{m_{r_{1}}^{i} e^{z_{1}^{i}}}\left[d_{x}+f\left(s_{11}^{i} z_{1}^{i}\right) \bar{d}_{1}\right]+\frac{s_{12}^{i} z_{1}^{i}}{m_{r_{1}}^{i} e^{z_{1}^{i}}}\left[d_{y}+f\left(s_{12}^{i} z_{1}^{i}\right) \bar{d}_{2}\right], \\
B & =\frac{s_{21}^{i} z_{2}^{i}}{m_{r_{2}}^{i} e^{z_{2}^{i}}}\left[d_{x}+f\left(s_{21}^{i} z_{1}^{i}\right) \bar{d}_{1}\right]+\frac{s_{22}^{i} z_{2}^{i}}{m_{r_{2}}^{i} e^{z_{2}^{i}}}\left[d_{y}+f\left(s_{22}^{i} z_{1}^{i}\right) \bar{d}_{2}\right], \\
C & =\left(\theta-\theta^{i *}\right)\left[d_{\theta}-f\left(\theta-\theta^{i *}\right) \bar{d}_{3}\right], \\
g_{11} & =s_{11}^{i}\left[v_{f}^{i} \cos \theta^{i *}-f\left(s_{11}^{i} z_{1}^{i}\right) \bar{d}_{1}\right], \\
g_{12} & =s_{12}^{i}\left[v_{f}^{i} \sin \theta^{i *}-f\left(s_{12}^{i} z_{1}^{i}\right) \bar{d}_{2}\right], \\
g_{21} & =s_{21}^{i}\left[v_{f}^{i} \cos \theta^{i *}-f\left(s_{21}^{i} z_{2}^{i}\right) \bar{d}_{1}\right], \\
g_{22} & =s_{22}^{i}\left[v_{f}^{i} \sin \theta^{i *}-f\left(s_{22}^{i} z_{2}^{i}\right) \bar{d}_{2}\right] .
\end{aligned}
$$

Select $\omega_{f}^{i}$ such that

$$
\left(\tan \phi-\tan \phi^{i *}\right)\left[\begin{array}{c}
\frac{\omega_{f}^{i}+\operatorname{sign}\left(\tan \phi-\tan \phi^{i *}\right) \bar{d}_{4}}{\cos ^{2} \phi} \\
-\frac{\dot{\phi}^{i *}}{\cos ^{2} \phi^{i *}}+\frac{v_{f}^{i}}{l}\left(\theta-\theta^{i *}\right)
\end{array}\right]<0,
$$

for all $\tan \phi \neq \tan \phi^{i *}$. One such a choice is given by

$$
\begin{aligned}
\omega_{f}^{i}= & \cos ^{2} \phi\left[\frac{\dot{\phi}^{i *}}{\cos ^{2} \phi^{i *}}-\gamma_{4}\left(\tan \phi-\tan \phi^{i *}\right)-\frac{v_{f}^{i}}{l}\left(\theta-\theta^{i *}\right)\right] \\
& -\operatorname{sign}\left(\tan \phi-\tan \phi^{i *}\right) \bar{d}_{4},
\end{aligned}
$$

where $\gamma_{4}>0$, which yields

$$
\begin{aligned}
\dot{L}^{i} \leq & -\gamma_{1}\left(z_{1}^{i}\right)^{2}-\gamma_{2}\left(z_{2}^{i}\right)^{2}-\gamma_{3}\left(\theta-\theta^{i *}\right)^{2} \\
& -\gamma_{4}\left(\tan \phi-\tan \phi^{i *}\right)^{2}+A+B+C .
\end{aligned}
$$

The next step is to prove that the terms $A, B$ and $C$ are upper bounded, which is done as follows. If $\left|s_{11}^{i} z_{1}^{i}\right| \geq \frac{\beta}{2 k} \ln \frac{\beta+1}{\beta-1}$, then $\frac{s_{11}^{i} z_{1}^{i}}{m_{r_{1}}^{i} e^{z_{1}^{i}}}\left[d_{x}+f\left(s_{11}^{i} z_{1}^{i}\right) \bar{d}_{1}\right] \leq 0$. Otherwise,

$$
\begin{aligned}
\frac{s_{11}^{i} z_{1}^{i}}{m_{r_{1}}^{i} e^{z_{1}^{i}}}\left[d_{x}+f\left(s_{11}^{i} z_{1}^{i}\right) \bar{d}_{1}\right] & \leq \frac{s_{11}^{i} z_{1}^{i}}{m_{r_{1}}^{i} e^{z_{1}^{i}}} d_{x} \\
& \leq-\frac{\left|s_{11}^{i}\right|}{m_{r_{1}}^{i}} \frac{\beta}{2 k\left|s_{11}^{i}\right|} \ln \frac{\beta+1}{\beta-1} \frac{\frac{\beta-1}{\beta+1}}{\frac{\beta}{2 k\left|s_{11}^{i}\right|}} \bar{d}_{1} \\
& <-\frac{\beta \bar{d}_{1}}{2 k m_{r_{1}^{i}}} \ln \frac{\beta+1}{\beta-1} e^{-\frac{\beta}{2 k\left|s_{11}^{i}\right|}}
\end{aligned}
$$

Therefore

$$
\begin{aligned}
& A \leq-\frac{\beta}{2 k m_{r_{1}}^{i}} \ln \frac{\beta+1}{\beta-1}\left(\bar{d}_{1} e^{-\frac{\beta}{2 k\left|s_{11}^{i}\right|}}+\bar{d}_{2} e^{-\frac{\beta}{2 k\left|s_{12}^{i}\right|}}\right)=M_{1}, \\
& B \leq-\frac{\beta}{2 k m_{r_{2}}^{i}} \ln \frac{\beta+1}{\beta-1}\left(\bar{d}_{1} e^{-\frac{\beta}{2 k\left|s_{21}^{i}\right|}}+\bar{d}_{2} e^{-\frac{\beta}{2 k\left|s_{22}^{i}\right|}}\right)=M_{2}, \\
& C \leq \frac{\beta d_{3}}{2 k} \ln \frac{\beta+1}{\beta-1}=M_{3} .
\end{aligned}
$$




$$
\begin{aligned}
& \tilde{\mathcal{R}}_{s}^{i}\left(v_{h}\right)=\left\{\left(m^{i}, \theta^{i}, \phi^{i}\right) \in \mathcal{Q}_{a}^{i} \times \mathbb{S} \times \mathbb{A}:\left(s_{j}^{i}\left[\cos \theta^{i}, \sin \theta^{i}\right]^{T} v_{h}\right) \leq \frac{1}{m_{j}^{i}+b_{2}}-\frac{1}{b_{2}} \text { if } m_{j}^{i} \geq-b_{2} \text { for all } j \in\{1,2\}\right\} \\
& \tilde{\mathcal{R}}_{h}^{i}\left(v_{h}\right)=\left\{\begin{array}{r}
\left(m^{i}, \theta^{i}, \phi^{i}\right) \in \mathcal{Q}_{a}^{i} \times \mathbb{S} \times \mathbb{A}: \quad \exists j \in\{1,2\} \text { such that }\left(s_{j}^{i}\left[\cos \theta^{i}, \sin \theta^{i}\right]^{T} v_{h}\right)>\frac{1}{m_{j}^{i}+b_{2}}-\frac{1}{b_{2}} \\
\text { and } m_{j}^{i} \geq-b_{2} \\
\text { and }\left(s_{k}^{i}\left[\cos \theta^{i}, \sin \theta^{i}\right]^{T} v_{h}\right)<\frac{1}{m_{k}^{i}+b_{1}}-\frac{1}{b_{1}} \text { if } m_{k}^{i} \geq-b_{1} \\
\text { for all } k \in\{1,2\}
\end{array}\right\} \\
& \left\{\begin{array}{r}
\left(m^{i}, \theta^{i}, \phi^{i}\right) \in \mathcal{Q}_{a}^{i} \times \mathbb{S} \times \mathbb{A}: \quad \exists j \in\{1,2\} \text { such that }\left(s_{j}^{i}\left[\cos \theta^{i}, \sin \theta^{i}\right]^{T} v_{h}\right) \geq \frac{1}{m_{j}^{i}+b_{1}}-\frac{1}{b_{1}}, \\
-b_{1} \leq m_{j}^{i}<0
\end{array}\right. \\
& \tilde{\mathcal{R}}_{d}^{i}\left(v_{h}\right)=\left\{\begin{array}{l}
\text { or } \exists j \in\{1,2\} \text { such that }\left(s_{j}^{i}\left[\cos \theta^{i}, \sin \theta^{i}\right]^{T} v_{h}\right)>\frac{1}{m_{j}^{i}+b_{1}}-\frac{1}{b_{1}},
\end{array}\right. \\
& m_{j}^{i}=0 \\
& \text { or } \left.\forall j \in\{1,2\} \text { such that } m_{j}^{i}=\left(s_{j}^{i}\left[\cos \theta^{i}, \sin \theta^{i}\right]^{T} v_{h}\right)=0\right\}
\end{aligned}
$$

Let $M=M_{1}+M_{2}+M_{3}$, then (25) indicates that

$$
\begin{aligned}
\dot{L}^{i} \leq & -\gamma_{1}\left(z_{1}^{i}\right)^{2}-\gamma_{2}\left(z_{2}^{i}\right)^{2}-\gamma_{3}\left(\theta-\theta^{i *}\right)^{2} \\
& -\gamma_{4}\left(\tan \phi-\tan \phi^{i *}\right)^{2}+M .
\end{aligned}
$$

Let $\gamma=\min \left\{\gamma_{1}, \gamma_{2}, \gamma_{3}, \gamma_{4}\right\}$, then

$$
\begin{aligned}
\dot{L}^{i} \leq & -\gamma\left[\left(z_{1}^{i}\right)^{2}+\left(z_{2}^{i}\right)^{2}+\left(\theta-\theta^{i *}\right)^{2}+\left(\tan \phi-\tan \phi^{i *}\right)^{2}\right] \\
& +M \\
= & -\gamma\left(L^{i}-\frac{M}{\gamma}\right),
\end{aligned}
$$

yielding $\lim _{t \rightarrow \infty}\left(L^{i}-\frac{M}{\gamma}\right)=0$. Therefore

$$
\begin{array}{r}
\lim _{t \rightarrow \infty}\left|z_{2}^{i}\right| \leq \sqrt{\frac{M}{\gamma \gamma_{2}}}, \quad \lim _{t \rightarrow \infty}\left|\tan \phi-\tan \phi^{i *}\right|<\sqrt{\frac{M}{\gamma \gamma_{4}}}, \\
\lim _{t \rightarrow \infty}\left|z_{1}^{i}\right| \leq \sqrt{\frac{M}{\gamma \gamma_{1}}}, \quad \lim _{t \rightarrow \infty}\left|\theta-\theta^{i *}\right|<\sqrt{\frac{M}{\gamma \gamma_{3}}} .
\end{array}
$$

The discussion in this section is summarized in the following statement.

Proposition 1: Consider the system (15) controlled by the feedback controller (22)-(24) with $m_{r}^{i}, \theta^{i *}$ and $\tan \phi^{i *}$ given by (18), (22) and (21a), respectively. Suppose the admissible Cartesian configuration set of the car $\mathcal{P}_{a}$ is described by (16) and $(x(0), y(0)) \in \mathcal{P}_{a}$. Then the closed-loop system has the following properties.

- $(x(t), y(t)) \in \mathcal{P}_{a}$ for all $t \geq 0$.

- Define the tracking errors as $e^{i}(t)=\left[e_{1}^{i}(t), e_{2}^{i}(t)\right]^{T}$ with

$$
e_{1}^{i}(t)=|| \frac{m_{1}^{i}(t)}{m_{r_{1}}^{i}(t)}|-1|, \quad e_{2}^{i}(t)=|| \frac{m_{2}^{i}(t)}{m_{r_{2}}^{i}(t)}|-1| .
$$

Then $\lim _{t \rightarrow \infty} e^{i}(t)$ is bounded. In addition, the bound can be controlled by tuning the parameters $\gamma_{1}, \gamma_{2}, \gamma_{3}, \gamma_{4}, \beta$ and $k$ of the feedback controller.
Proof: According to the definition of $z^{i}$ given by (17), $m_{j}^{i}(t)<0$ for all $t \geq 0$ and $j \in\{1,2\}$. This implies the first claim.

Consider the Lyapunov function (23) and note that (26) holds with the help of the feedback controller (22)-(24). This implies

$$
e^{-\sqrt{\frac{M}{\gamma \gamma_{1}}}} \leq \frac{m_{1}^{i}}{m_{r_{1}}^{i}} \leq e^{\sqrt{\frac{M}{\gamma \gamma_{1}}}}
$$

yielding

$$
e_{1}^{i}(t) \leq \min \left(\left|e^{\sqrt{\frac{M}{\gamma \gamma_{1}}}}-1\right|,\left|1-e^{-\sqrt{\frac{M}{\gamma \gamma_{1}}}}\right|\right) .
$$

Hence, the second statement holds.

\section{B. Robust Shared-Control Algorithm}

Similarly to the previous section we need to determine the safe subset $\mathcal{R}_{s}$ before providing the shared-control law. Relative to each human input and the $i^{\text {th }}$ group of constraints, the overall set can be partitioned into three subsets: the safe subset $\tilde{\mathcal{R}}_{s}$, the hysteresis subset $\tilde{\mathcal{R}}_{h}$ and the dangerous subset $\tilde{\mathcal{R}}_{d}$ as described by equation (27) at the top of this page, where $\mathcal{Q}_{a}^{i}=S^{i} \mathcal{P}_{a}+$ $T^{i}$, and $b_{2}>b_{1}>0$. The intuition of this definition is similar to that for (12). Note that the definition of three subsets defined by (26) is given in the $\left(m^{i}, \theta^{i}, \phi^{i}\right)$ coordinates and can be transfered into the $(x, y, \theta, \phi)$ coordinates by

$$
\begin{aligned}
& \mathcal{R}_{s}^{i}\left(v_{h}\right)=\operatorname{diag}\left(\mathrm{S}^{\mathrm{i}}-1, \mathrm{I}\right)\left(\tilde{\mathcal{R}}_{\mathrm{s}}^{\mathrm{i}}-\operatorname{col}\left(\mathrm{T}^{\mathrm{i}}, 0\right)\right), \\
& \mathcal{R}_{h}^{i}\left(v_{h}\right)=\operatorname{diag}\left(\mathrm{S}^{\mathrm{i}}-1, \mathrm{I}\right)\left(\tilde{\mathcal{R}}_{\mathrm{h}}^{\mathrm{i}}-\operatorname{col}\left(\mathrm{T}^{\mathrm{i}}, 0\right)\right), \\
& \mathcal{R}_{d}^{i}\left(v_{h}\right)=\operatorname{diag}\left(\mathrm{S}^{\mathrm{i}-1}, \mathrm{I}\right)\left(\tilde{\mathcal{R}}_{\mathrm{d}}^{\mathrm{i}}-\operatorname{col}\left(\mathrm{T}^{\mathrm{i}}, 0\right)\right),
\end{aligned}
$$

where $\operatorname{col}\left(T^{\mathrm{i}}, 0\right)$ is a column vector obtained by stacking the zero vector under the vector $T^{i}$. 
Note that in the cases in which two constraints are active, there is only one group of active constraints. However, if only one constraint is active, there could be $n_{c}$ groups of active constraints, where $n_{c} \leq m-1$. Therefore, the activation of the $i^{\text {th }}$ group of constraints is not equivalent to the activation of the $i^{\text {th }}$ feedback controller $u_{f}^{i}$. Suppose $I_{1}, I_{2}, \ldots, I_{n_{c}}$ groups of constraints are active at the time instant $t$, where $I_{i} \in\left\{1,2, \ldots, N_{c}\right\}$ for all $i \in\left\{1,2, \ldots, n_{c}\right\}$ and $L^{j}$ is defined in equation (23). Then the sharing function $k^{i}$ relative to the $i^{\text {th }}$ group of constraints is defined as

$$
k^{i}\left(p, \theta, v_{h}, t\right)= \begin{cases}0, & (p, \theta, \phi) \in \mathcal{R}_{d}^{i} \text { and } L^{i}=\min _{j=I_{1}}^{I_{n_{c}}} L^{j}, \\ g^{i}, & (p, \theta, \phi) \in \mathcal{R}_{h}^{i}, \\ 1, & \text { otherwise, }\end{cases}
$$

where

$$
g^{i}= \begin{cases}0, & \text { if } k^{i}\left(t^{-}\right)=0 \\ 1, & \text { if } k^{i}\left(t^{-}\right)=1\end{cases}
$$

On the basis of three subsets, the shared-control algorithm is given as

$$
\begin{aligned}
u_{s}\left(p, \theta, \phi, v_{h}\right)= & \sum_{i=1}^{N_{c}}\left[\left(1-k^{i}\left(p, \theta, v_{h}\right)\right) u_{f}^{i}\left(p, \theta, \phi, p_{r}, \theta_{r}, \alpha_{r}\right)\right] \\
& +\min _{i=1}^{N_{c}} k^{i}\left(p, \theta, v_{h}\right) u_{h} .
\end{aligned}
$$

Proposition 2: Consider the kinematic model of a rear-wheel drive car with disturbances given in equation (15) and controlled by the shared-controller (22)-(24)-(28)-(29). Suppose the admissible configuration set of the car $\mathcal{P}_{a}$ is a non-empty and compact set defined by (16) and $(x(0), y(0)) \in \mathcal{P}_{a}$. Let $u_{h}$ be a given h-control such that the h-closed-loop system is stable. Then there exist positive constants $\gamma_{1}, \gamma_{2}, \gamma_{3}$ and $b_{2}>b_{1}>0$ such that the s-closed-loop system has the following properties.

1) $(x(t), y(t)) \in \mathcal{P}_{a}$ for all $t \geq 0$.

2) The tracking error for the s-closed-loop system is bounded.

3) $u_{s}(t)=u_{h}(t)$ for all $(p(t), \theta(t), \phi(t)) \in \mathcal{R}_{s}\left(v_{h}(t)\right) \backslash$ $\mathcal{R}_{d}\left(v_{h}(t)\right)$ and $t \geq 0$.

Proof: The proof is similar to that of Theorem 1, hence omitted.

\section{CASE STUDY}

This section discusses two case studies: traffic at a five-way junction and at a roundabout, to demonstrate the effectiveness of the shared-control algorithm. Further examples, such as emergency braking and overtaking on highways, can be found in [27], [28]. We assume the projection of the car in $(x, y)$ plane is a rectangle with size $1 m \times 1.5 m$.

\section{A. Traffic at a Five-Way Junction}

In the case study we use the name SE-Road, N-Road and W(E)-Road to denote the road heading to the South-east, the North and the West (East), respectively. Note that the N-Road is a one-way road and the SE-Road is a side road while all the other roads are main roads. We assume that the traffic on the N-Road has a higher priority over that on the W(E)-Road. The scenario is simulated by MATLAB SIMULINK and the results show that the safety of the car with shared-controller is guaranteed even if the driver behaves dangerously.

Simulation results are displayed in Fig. 4, which show a sequence of snapshots at the junction. There are 6 cars with three different colors, representing different priorities, in Fig. 4 (a). The three green cars have the highest priority. The one on the W(E)-Road is going to turn left at the junction and the other two on the N-Road keep driving straight when they approach the junction. The two blue cars have the second highest priority and they are heading to the East and West, respectively. The yellow car has the lowest priority and is driven to the side road, the SE-Road. After a while the feedback controller for the blue car heading to the West is active and gives way to the green cars on the N-Road as indicated by sub-figure (b) of Fig. 4: the color of the car changes to red. Then the feedback controllers for the blue car heading to the East and the yellow car are active one after the other as shown in Fig. 4 (c) and (d). The control authorities of the car are passed to the drivers of the blue cars when the two green cars on the N-Road pass the junction, while the yellow car should wait until all the other cars pass the junction, since it has the lowest priority as displayed in Fig. 4 (e) to (g). In conclusion, six cars negotiate the junction safely with the help of the shared-controller as indicated by Fig. 4 . Note that the feedback controller is active not only to stop the car but also to assist steering ( $\omega_{s}$ is used to control the steering angle).

\section{B. Traffic at a Roundabout}

This case studies the traffic at a roundabout where the car should give way to vehicles coming from the right-hand side. We use the name N-Exit, E-Exit, S-Exit and W-Exit to denote the exit at the North, the East, the South and the West of the roundabout, respectively. We assume that the controlled car is coming from the S-Exit and aims to turn right to leave the roundabout from the E-Exit. Simulation results are displayed in Fig. 5, in which 4 snapshots are given to show how the traffic moves at the roundabout. Before the controlled car entering the roundabout, one blue car is leaving the E-Exit and heading to the N-Exit as shown in Fig. 5 (a). However, the driver of the controlled car does not recognize it and keeps driving to the roundabout. Hence, the feedback controller is active (represented by the red rectangle in Fig. 5 (b)) to stop the car. When the right of the controlled car is clear, the driver regains the control authority and enters the roundabout. However, the driver does not turn properly. Therefore the feedback controller is active again to assist the driver and to make sure that the car goes to the right lane as displayed in 


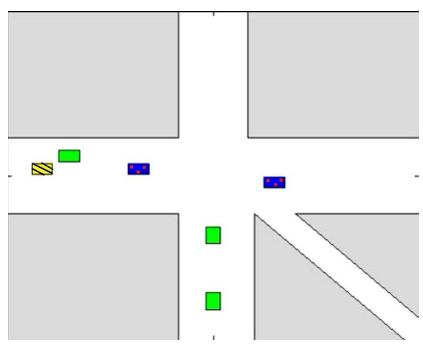

(a)

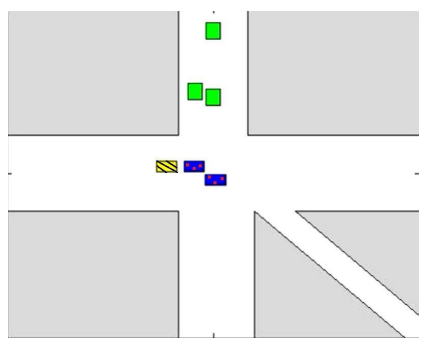

(e)

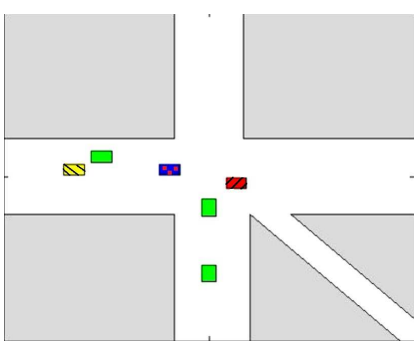

(b)

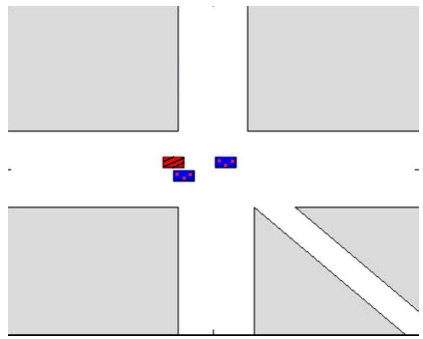

(f)

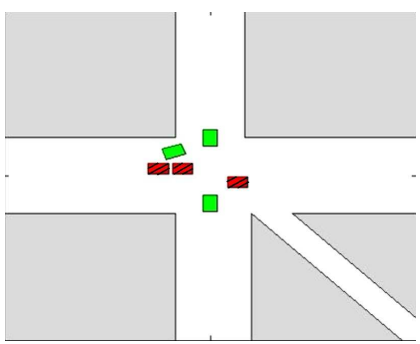

(c)

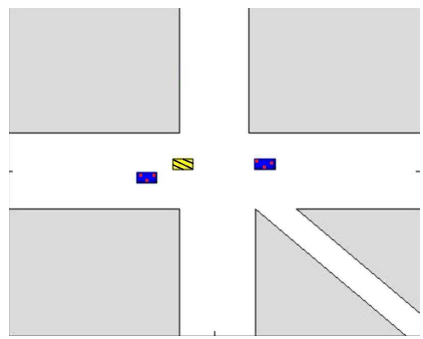

(g)

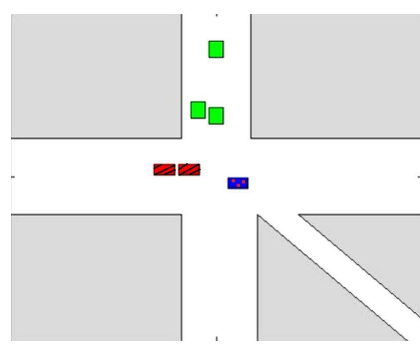

(d)
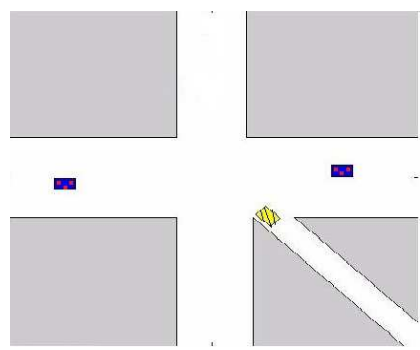

(h)

Fig. 4: Snapshots at the junction displaying paths of the cars with the shared-control in the $(x, y)$-plane for the set $\mathcal{P}_{a}$ represented by the white area. Green cars: the controlled cars (the feedback controller is not active) with the highest priority. Blue dotted cars: the controlled cars (the feedback controller is not active) with the second highest priority. Yellow backslashed car: the controlled car (the feedback controller is not active) with the lowest priority. Red slashed cars: the controlled cars (the feedback controller is active).

Fig. 5 (c). Finally, when the car is safe the control authority is passed back to the driver.

Note that the simulation results refers to the case when the human driver behaves "dangerously" and the feedback controllers are active, indicated by red colors. This 'red' signal is fed back to the human driver, implying that he/she does not operate safely. The control authority can be regained by the driver if he/she operates properly.

\section{CONCLUSIONS}

We have developed a solution to the shared-control problem for the kinematic model of a rear-wheel driving car without measurements of absolute positions. The solution can be used to deal with static and dynamic feasible Cartesian position sets of the car. We have added disturbances to the dynamics and prove that if all the disturbances are bounded then the $(x, y)$-trajectory of the car is able to track a feasible reference with bounded errors. The shared-control algorithm integrates the human input and the feedback control input based on a hysteresis switch and the control authority is held by the driver most of the time but it is gained by the feedback controller in "dangerous" situations. Since the feedback controller is not active all the time, there is no need to model and predict the human behaviour. Instead, we measure and monitor human inputs all the time and the feedback controller is active only if system states belong to the dangerous subset $\mathcal{R}_{d}$. The case studies given in Section $\mathrm{V}$ demonstrates the effectiveness of the shared-control law. Future work will focus on systems with measurement errors and cooperations among controlled cars.

\section{REFERENCES}

[1] C. Gomes, Global auto report . Scotiabank Economics.

[2] WHO, Global Status Report on Road Safety 2013: Supporting a Decade of Action. Luxemourg.

[3] C. Hwang and L. Chang, "Trajectory tracking and obstacle avoidance of car-like mobile robots in an intelligent space using mixed $\mathrm{h} 2 / \mathrm{h}$ decentralized control," IEEE Trans. on Mechatronics, vol. 12, no. 3, pp. 345-352, 2007.

[4] D. Corona and B. De Schutter, "Adaptive Cruise Control for a SMART Car: A Comparison Benchmark for MPC-PWA Control Methods," IEEE Trans. on Control Systems Technology, vol. 16, no. 2, pp. 365-372, 2008.

[5] A. Furda and L. Vlacic, "Towards increased road safety: Real-time decision making for driverless city vehicles," in Proc. of IEEE Conference on Systems, Man and Cybernetics, 2009, pp. 2421-2426.

[6] C. Hwang, L. Chang, and Y. Yu, "Network-based fuzzy decentralized sliding-mode control for car-like mobile robots," IEEE Trans. on Industrial Electronics, vol. 54, no. 1, pp. 574-585, 2007.

[7] N. Yagiz, Y. Hacioglu, and Y. Taskin, "Fuzzy sliding-mode control of active suspensions," IEEE Trans. on Industrial Electronics, vol. 55, no. 11 , pp. $3883-3890,2008$.

[8] N. Ghani and Y. Sam, "Sliding mode control of active car steering with various boundary layer thickness and disturbances," in Proc. of IEEE Conference on Industrial Electronics and Applications, 2007, pp. 24942499.

[9] J. Lin and R. Lian, "Intelligent control of active suspension systems," IEEE Trans. on Industrial Electronics, vol. 58, no. 2, pp. 618-628, 2011.

[10] L. Ellis, N. Pugeault, K. Ofjall, J. Hedborg, R. Bowden, and M. Felsberg, "Autonomous navigation and sign detector learning," in Proc. of IEEE Workshop on Robot Vision, 2013, pp. 144-151.

[11] D. Ho and J. Garibaldi, "A novel fuzzy inferencing methodology for simulated car racing," in Proc. of IEEE International Conference on Fuzzy Systems, 2008, pp. 1907-1914. 


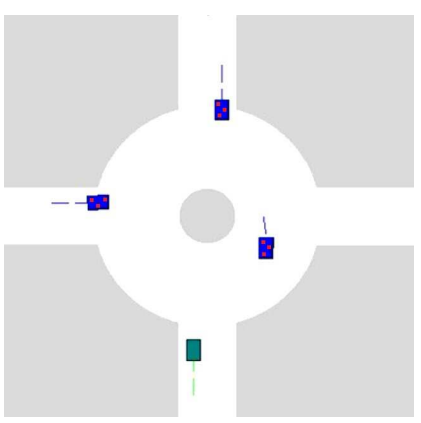

(a)

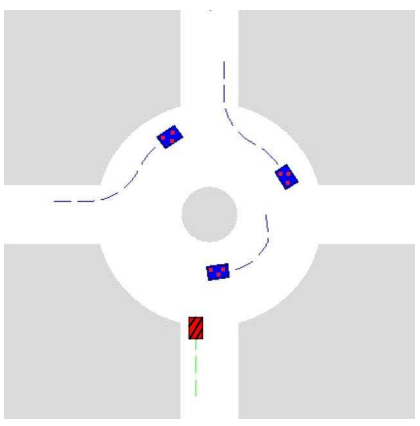

(b)

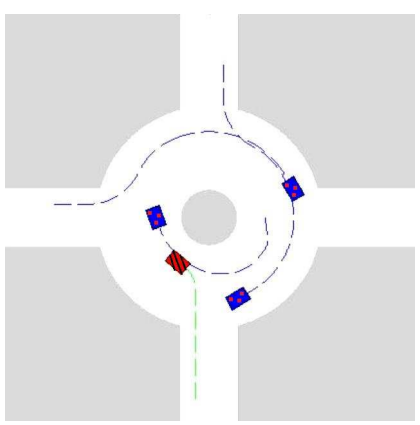

(c)

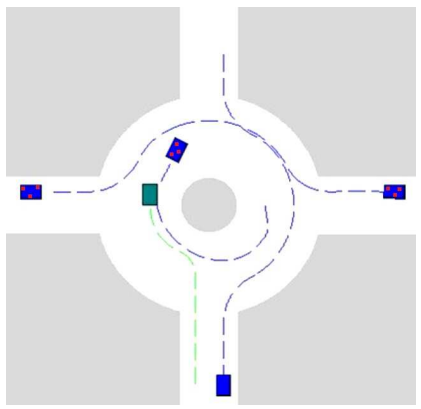

(d)

Fig. 5: Snapshots at the roundabout displaying paths of the cars with the shared-control in the $(x, y)$-plane for the set $\mathcal{P}_{a}$ represented by the white area. Green car: the controlled cars (the feedback controller is not active). Red slashed car: the controlled cars (the feedback controller is active). Blue dotted cars: other vehicles at the roundabout. Green dashed line: $(x, y)$-trajectory of the controlled car. Blue dashed lines: $(x, y)$-trajectory of the other cars on the road.

[12] I. Baturone, F. Moreno-Velo, S. Sanchez-Solano, and A. Ollero, "Automatic design of fuzzy controllers for car-like autonomous robots," IEEE Trans. on Fuzzy Systems, vol. 12, no. 4, pp. 447-465, 2004.

[13] T. Li and S. Chang, "Autonomous fuzzy parking control of a car-like mobile robot," IEEE Trans. on Systems, Man and Cybernetics, Part A: Systems and Humans, vol. 33, no. 4, pp. 451-465, 2003.

[14] C. Hwang and L. Chang, "Internet-based smart-space navigation of a car-like wheeled robot using fuzzy-neural adaptive control," IEEE Trans. on Fuzzy Systems, vol. 16, no. 5, pp. 1271-1284, 2008.

[15] A. Bejczy, "The effects of level of automation and adaptive automation on human performance, situation awareness and workload in a dynamic control task," Theoretical Issues in Ergonomics Science, vol. 5, no. 2, pp. 113-153, 2004.

[16] J. Alonso-Mora, P. Gohl, S. Watson, R. Siegwart, and P. Beardsley, "Shared control of autonomous vehicles based on velocity space optimization," in Proc. of IEEE Conference on Robotics and Automation, 2014, pp. 1639-1645.

[17] A. Franchi, C. Secchi, M. Ryll, H. Bulthoff, and P. Giordano, "Shared Control : Balancing Autonomy and Human Assistance with a Group of Quadrotor UAVs," IEEE Robotics and Automation Magazine, vol. 19, no. 3, pp. 57-68, 2012.

[18] T. Sheridan and R. Parasuraman, "Human-automation interaction," Journal of Human Factors and Ergonomics, vol. 1, no. 1, pp. 89-129, 2005.

[19] M. Hafner, D. Cunningham, L. Caminiti, and D. Vecchio, "Cooperative collision avoidance at intersections: Algorithms and experiments," IEEE Trans. on Intelligent Transportation Systems, vol. 14, no. 3, pp. 1162 1175,2013

[20] S. Hurley, "The shared circuits model (SCM): How control, mirroring, and simulation can enable imitation, deliberation, and mindreading," Behavioral and Brain Sciences, vol. 31, no. 1, pp. 1-22, 2008.

[21] P. Cacciabue, Modelling and Simulation of Human Behaviour in System Control. Springer Publishing Company, 2011.

[22] L. Suchman, Human-machine reconfigurations: Plans and situated actions. Cambridge University Press, 2007.

[23] J. Jiang and A. Astolfi, "Shared-control for the kinematic model of a rear-wheel drive car," in Proc. of American Control Conference, Chicago, USA, 2015.

[24] C. Byrnes, F. Celani, and A. Isidori, "Omega-limit sets of a class of nonlinear systems that are semiglobally practically stabilized," International Journal of Robust and Nonlinear Control, vol. 15, no. 7, pp. 315-333, 2005.

[25] J. Jiang and A. Astolfi, "State and output-feedback shared-control for a class of linear constrained systems," IEEE Trans. on Automatic Control, vol. PP, no. 99,2016
[26] C. Prieur and A. Teel, "Uniting local and global output feedback controller," IEEE Trans. Automatic Control, vol. 56, no. 7, pp. 16361679,2011

[27] Web link: shared control, https://youtu.be/nwvOlewt65U.

[28] J. Jiang, "Shared-control for systems with constraints," Ph.D. dissertation, Imperial College London, Department of Electrical and Electronic Engineering, Imperial College London, London, UK. SW7 2AZ, 2016.

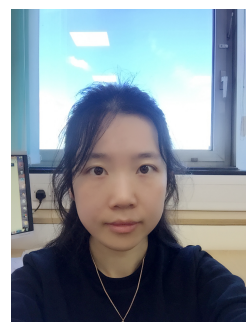

Jingjing Jiang was born in China. She received the B.Eng. Degree in Electrical and Electronic Engineering from the University of Birmingham, UK, and the Harbin Institute of Technology, China, in 2010, the M.Sc. degree in Control Systems with Department Prize for Outstanding Achievement from Imperial College London, UK, in 2011, and the Ph.D degree from Imperial College London, in 2016, with a Thesis on Shared-Control for Systems with Constraints. Since 2016 she has been with the Electrical and Electronic Engineering Department of Imperial College London, London (UK), where she is currently a research associate in the Control and Power Group. Her current research interests include control design of systems with constraints, human-in-the-loop, driver assistance control and autonomous motorcycle control design.

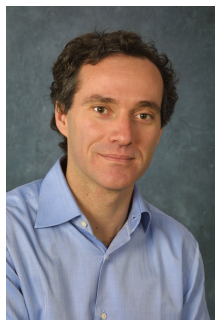

Alessandro Astolfi (F', 2009) was born in Rome, Italy. He received the Bachelor Degree in Electrical Engineering from the University of Rome, Rome, in 1991, the M.Sc. in Information Theory and the Ph.D. (Hons.) with a Thesis on Discontinuous Stabilization of Nonholonomic Systems from ETH Zurich, Zrich, Switzerland, in 1995, and the Ph.D. degree on nonlinear robust control from the University of Rome in 1996. He has been with the Electrical and Electronic Engineering Department, Imperial College London, London, UK., since 1996, where he is currently a Professor of Nonlinear Control Theory and the Head of the Control an Power Group. He was an Associate Professor with Politecnico of Milano, Milan, Italy, from 1998 to 2003. He has also been a Professor at University of Rome Tor Vergata, Rome, since 2005. His current research interests include control theory and applications, with special emphasis for the problems of discontinuous stabilization, robust and adaptive control, observer design and model reduction. Dr. Astolfi, a Distinguished Member of the IEEE Control Systems Society (CSS), was a recipient of the IEEE CSS A. Ruberti Young Researcher Prize in 2007, and the IEEE CSS George S. Axelby Outstanding Paper Award in 2012. 